\title{
A Practical Approach to Anaesthesia for the Child with Musculoskeletal Disease
}

\author{
Kay J \\ Private Practice Anaesthesia \\ Corresponding author, email: info@drjkay.co.za
}

This article aims to provide the reader with a practical and brief approach to managing or avoiding the many issues associated with anaesthesia for children with a muscular or skeletal abnormality or disease.

The disorders mentioned in this note are some of the more common to present for anaesthesia management. They are not comprehensively described below, instead, the major areas of concern for anaesthesia providers are set down in each case.

\section{Cerebral Palsy}

Cerebral palsy is a non-progressive encephalopathy which manifests as varying degrees of motor, sensory and intellectual deficit. It is estimated to affect approximately 1 in 500 live births and (contrary to much belief) is related to an intra-uterine event during foetal development in around $80 \%$ of cases. Of all cases of CP identified, birth complications make up less than $10 \%$ of all causes and no cause is ever identified in around $30 \%$ of cases.

Most children suffering from $\mathrm{CP}$ will have an increase in muscle tone and much of the surgical work done involves mitigating the effects of spasticity. ${ }^{1}$

\section{Main Areas of Concern}

\section{Neurological System and Sensation}

- Many CP children have impaired vision or hearing. Cognitive deficits are common, and they are frequently unable to understand or make themselves understood. They require sensitive and compassionate handling and bright lights, rapid changes in environment, loud noises and large groups of people are to be avoided.

- Spastic posturing often makes for difficult IV access; scoliosis similarly complicates regional anaesthesia.

- Many of these children are epileptic and are typically quite brittle. The omission of medications prior to surgery is to be avoided.

\section{Gastrointestinal, Nutritional and Respiratory}

- CP children frequently manifest difficulties with swallowing and gastro-oesophageal reflux which complicate their feeding. They are prone to clinical or subclinical aspiration and often have poor respiratory function and frequent chest infections.

- Another result of the poor feeding performance is that they are frequently undernourished (despite the best efforts of the caregivers) resulting in low muscle and body fat bulk. Care must be taken to ensure adequate warming during anaesthesia, and careful history around feeding as well as current respiratory health must be taken.

- Finally, a tendency to more bleeding is frequently mentioned, particularly in the severe malnourished CP child. Care and attention to the application of tourniquet where applicable and vigilance for uncontrolled haemorrhage is important.

\section{Polypharmacy}

- CP children are frequently on a number of medications which may include anticonvulsants, antispasticity medications, antireflux medications and even behavioural modifiers. Drug interactions should be carefully considered.

- Anaesthesia drug effects are unpredictable. Children with severe deficits and those receiving anticonvulsants may have a considerably decreased MAC. Chronic respiratory disease may increase sensitivity to depressant medications, such as opiates and sedatives. ${ }^{2}$

\section{Dwarfism}

Children with abnormally short stature frequently present for surgery either related or unrelated to their underlying problem. Achondroplasia is the commonest heritable form of dwarfism and affects around 1:7500 individuals. ${ }^{3}$

\section{Main Areas of Concern}

\section{Airway Management}

- All dwarfism is noted to present an airway of increased difficulty ranging from mildly increased in the case of achondroplasia to massively difficult in the case of the mucopolysaccharidoses.

- Abnormalities include abnormal facial anatomy causing poor mask fit, macroglossia and micrognathia, tracheal stenosis and abnormal laryngeal anatomy.

- Great care must be taken in examining the airway and planning airway management ahead of time. A second pair of experienced hands to assist will also be essential should more advanced manoeuvres prove necessary.

- Instability of the cervical spine features prominently in dwarfism and as little as possible manipulation should be performed. 


\section{Sleep Apneoa and Respiratory Function}

- Most forms of dwarfism suffer from some degree of sleep apnoea. In the case of achondroplasia, this is thought to be related to pressure as a result of the smaller than usual space afforded the medulla and is of the central apnoea type. In other forms, macroglossia and abnormal airway anatomy predispose to the usual obstructive type sleep apnoea.

- In both cases, care must be taken when dosing medications which worsen sleep apnoea.

\section{Skin, MSK and regional}

- Achondroplasia may cause excess thick skin which may make IV access difficult.

- Careful positioning may be necessary to avoid the formation of abnormal pressure points.

- Regional anaesthesia is an attractive option in these patients, however titratable methods such as epidural are favoured, and while peripheral regional anaesthesia is certainly possible, anatomy may make this route considerably more challenging than usual. ${ }^{4}$

\section{Osteogenesis Imperfecta}

Osteogenesis imperfecta is the commonest heritable skeletal dysplasia and affects around 1 in every 20000 people. Most OI involves some aberration in the formation of type I collagen.

\section{Main Areas of Concern}

\section{Airway and Handling}

- As with the dwarfism syndromes, considerable care should be exercised when manipulating the cervical spine and jaw as they are prone to dislocation and fractures.

- All joints are prone to subluxation and bones can fracture with minimal force. Great care must be taken when transferring to and from the operating table and padding pressure points.

\section{Cardiac anomalies}

- As collagen is the foundation of the fibrous cardiac skeleton, cardiac disease has an increased frequency in OI. Valve regurgitation is common as well as dilatation of the aortic root. These problems are infrequent in children with the disorder, however.

\section{Bleeding}

- Platelet related bleeding and clotting factor deficiencies have both been observed in patients with OI. Avoidance of anticoagulant medications seems sensible.

- Cryoprecipitate, factor concentrates and platelet infusions have all been tried with some success.

\section{Hyperthermia}

- Another reported complication of anaesthesia in OI is the "malignant hyperthermia-like" syndrome of hyperthermia and hypermetabolism.

- While there is not an association between true $\mathrm{MH}$ and $\mathrm{OI}$ there is an increased propensity of these patients to manifest in a similar fashion.
- Pathogenesis of this feature is unknown but suggested mechanisms involve abnormal central temperature regulation, abnormal muscle metabolism (CK levels are raised in patients with this problem) and a potential link to abnormal thyroid function (elevated thyroid hormone concentrations in $50 \%$ of OI).

- Current evidence supports the avoidance of $\mathrm{MH}$ trigger agents, and treatment as for malignant hyperthermia seems reasonable if this complication arises. ${ }^{5}$

\section{Muscle Disorders}

This section is potentially larger than all the sections which have come before in this article. Each of the disorders mentioned below has its own peculiarities and particular features, symptoms and signs. In the interests of applicability, however, they will be treated together as a group to give a unified "catch-all" approach to the management of a child with a suspected or known muscle disorder.

This section includes a practical guideline for the following muscle disorders:

- Beckers and Duchenne muscular dystrophies (dystrophinopathies)

- Congenital myopathies (Central core, mitochondrial, multicore, mini-core and nemaline rod)

\section{- Myotonias (Dystrophic and Congenital type) ${ }^{6}$}

The following points are appropriate in all children:

1. Children with no previous medical history must be regarded as potentially having a muscle disorder and the features which are suggestive must be actively sought. Table 1 includes common findings which should raise suspicion of a muscle disorder.

2. Children with a known muscle disorder will frequently not be able to name their disorder, nor will their parents.

3. A serum creatine kinase is extremely useful in guiding management of these cases

4. Muscle diseases almost always affect the cardiac and respiratory systems, and energy metabolic processes are frequently also involved.

Table 1 - Clinical signs and symptoms associated with muscle disease, adapted from Brandom, B (2013)

\begin{tabular}{ll}
\hline Family History & Muscle disease in the family \\
Personal History & Hypotonia \\
& $\begin{array}{l}\text { Arthrogryposis } \\
\text { Delayed motor development } \\
\text { Painful muscle cramps } \\
\text { Dark urine following exercise or anaesthesia }\end{array}$ \\
& $\begin{array}{l}\text { Firm enlarged calves } \\
\text { Examination }\end{array}$ \\
& $\begin{array}{l}\text { Amyotrophy } \\
\text { Myopathic facies } \\
\text { Walking like a duck }\end{array}$ \\
& Tachycardia at rest \\
Investigations & $\begin{array}{l}\text { Asymptomatic elevated CK or AST } \\
\text { ECG - conduction abnormality }\end{array}$ \\
\hline
\end{tabular}




\section{Main Areas of Concern}

\section{Potential for malignant hyperthermia or anaesthesia-induced rhabdomyolysis}

- It should be noted that of children who manifest a hyperpyrexial or rhabdomyolytic episode under anaesthesia, less than $10 \%$ give a family history of such an issue and $50 \%$ of such patients have undergone at least 2 previous anaesthetics with trigger agents without incident. Such history is, therefore, no guarantee of safety.

- It is reasonable that all children with a known or suspected muscle disease should undergo a trigger agent free anaesthetic with a clean anaesthetic machine. This should include all children with an elevated serum CK unless a plausible alternative cause is found.

- It is reasonable that all children with any form of known or suspected muscle disease should NOT receive suxamethonium.

- Unless the child in question is known to have a condition that is strongly associated with either AIR or MH. A gas induction with sevoflurane is reasonable before converting to a total intravenous technique.

- Anaesthesia providers should familiarise themselves with the appropriate action should an attack of MH/AIR be suspected. In particular, surgery must be terminated, dantrolene administered, intensive care informed. As little as possible delay should be allowed before critical care is instituted.

- Temperature management should aim for normothermia at all times. It is tempting to avoid active warming in order to aid in identifying hyperpyrexia, but low temperatures are harmful and particularly so in myotonias where they reliably precipitate spasms.

\section{Cardiovascular Disease}

- As mentioned above, cardiovascular and respiratory complications are frequently encountered in these children.

- Cardiac manifestations can range from abnormally high resting heart rate to full-blown cardiac failure and arrhythmias.

- Cardiac disease can occasionally be more marked than the accompanying muscle disease.

- Avoidance of negatively inotropic drugs and a low threshold for CVS support is recommended.

- Dystrophinopathies are particularly notable for their CVS manifestations and this can even include female carriers of the gene (who also have an elevated CK).

\section{Respiratory Compromise}

- As a result of muscle weakness involving the respiratory system, these children manifest frequent chest infections and may have a poor cough.

- Subclinical aspiration due to poor pharyngeal muscle function has been seen. Sleep apnoea is also a consideration.

- Preoperative workup should include chest X-rays, pulmonary functions as appropriate and night oximetry to detect sleep apnoea if suspected.

- Postoperative ventilatory support should be considered in major surgery or severely affected individuals

\section{Other systems}

- Gastrointestinal tract issues are also frequently seen with most of the diseases mentioned above which may complicate postoperative nutrition.

- Carbohydrate intake and energy balance must be carefully controlled particularly in the mitochondrial myopathies and periodic paralyses.

- Muscle disorders are associated with dysmorphisms and other congenital abnormalities which should be considered, particularly the challenges they might add to airway management. ${ }^{7,8}$

In conclusion, musculoskeletal disorders are an inhomogeneous group of conditions, each of which brings distinct challenges to the anaesthesia provider. Although far from comprehensive, it is the author's hope that this note will serve as a useful guide in the practical management of these children during the many anaesthetics they will certainly require in their lifetime.

\section{References}

1. Prosser D, Sharma N. Cerebral palsy and anaesthesia. Continuing Education in Anaesthesia Critical Care \& Pain. 2010;10(3):72-6.

2. Wilton N, Anderson B. Orthopedic and Spine Surgery. In: Coté C, Lerman J, Todres I. A practice of anesthesia for infants and children. 4th ed. Philadelphia Pa.: Saunders/Elsevier; 2009. p. 651-5.

3. Ramani R. Skin and Musculoskeletal Diseases. In: Hines R, Marschall K. Stoelting's anesthesia and co-existing disease. 6th ed. Philadelphia: Elsevier; 2012. p. 459-63.

4. Zuckerberg AL, Yaster M. Anesthesia for Pediatric Orthopedic Surgery. In: Davis P, Cladis F. Smith's anesthesia for infants and children. 9th ed. St Louis: Elsevier; 2017. p. 865-91.

5. Reece L. Anesthetic implications for the patient with osteogenesis imperfecta. AANA journal. 2010;78(1):47.

6. Marsh S, Pittard A. Neuromuscular disorders and anaesthesia. Part 2: specific neuromuscular disorders. Continuing Education in Anaesthesia Critical Care \& Pain. 2011;11(4):119-23.

7. Brandom B, Veyckemans F. Neuromuscular diseases in children: a practica approach. Pediatr Anesth. 2013;23(9):765-9.

8. Sivak E, Brandom B. Malignant Hyperthermia. In: Davis P, Cladis F. Smith's anesthesia for infants and children. 9th ed. St Louis: Elsevier; 2017. p. 1188-200. 